\title{
Descriptive epidemiology of type 1 diabetes-is it still in?
}

\author{
M. Knip
}

Received: 5 July 2011 / Accepted: 22 September 2011 / Published online: 9 March 2012

(C) Springer-Verlag 2012

\begin{abstract}
This edition of 'Then and now' discusses the valuable contribution made by Onkamo and colleagues to the field of type 1 diabetes epidemiology in their widely cited paper 'Worldwide increase in incidence of type I diabetes-the analysis of the data on published incidence trends', which was published 13 years ago (Diabetologia 1999;42:1395-1403). At the time, this represented the most extensive analysis of global trends in the epidemiology of type 1 diabetes, and covered/included a considerably larger geographical area than previous studies. The data confirmed that there was a worldwide increase in the incidence of childhood diabetes during the second half of the 20th century. Predictions made by the group for the incidence rates in 2010 pointed to large increases, but in retrospect these turned out to be too conservative, particularly among younger children. Whether the increase in incidence among children aged $<15$ years has started to level off is unknown. Looking to the future, more data on the epidemiology of type 1 diabetes over the whole lifespan are definitely needed.
\end{abstract}

\footnotetext{
M. Knip ( $\bowtie)$

Children's Hospital, University of Helsinki,

P.O. Box 22, FI-00014 Helsinki, Finland

e-mail: mikael.knip@helsinki.fi

M. Knip

Children's Hospital, Helsinki University Central Hospital,

Helsinki, Finland

M. Knip

Folkhälsan Research Center,

Helsinki, Finland

M. Knip

Department of Pediatrics, Tampere University Hospital,

Tampere, Finland
}

In addition, descriptive epidemiology needs to be complemented with 'aetiological' epidemiology generating information on the causes of the incidence and prevalence trends.

Keywords Age · Children · Environment · Epidemiology Finland · Genes · Incidence · Registers · Type 1 diabetes

Type 1 diabetes is an immune-mediated disease that develops as a consequence of the gradual destruction of the insulin-producing beta cells, which eventually results in total beta cell loss and complete dependence on exogenous insulin. The disease may present at any age, but the majority of patients are diagnosed before the age of 30 years. The disease process is initiated months and years before the emergence of clinical symptoms [1]. The epidemiology of type 1 diabetes has been best characterised in children and adolescents under the age of 15 years.

In 1999 Onkamo et al. published an article on global incidence trends in type 1 diabetes from 1960 to 1996 based on a systematic literature review [2]. At that time they identified 160 original publications reporting time series of the incidence of type 1 diabetes. They ended up with 37 studies from 27 countries that fulfilled the preselected inclusion criteria: (1) a study period of 8 years or more; (2) incidence rates presented separately for each year; (3) five or more cases per year; (4) in the papers in which the age standardisation had been reported, the incidences were estimated with age standardisation according to the world population; and (5) type 1 diabetes diagnosed according to the WHO definition. The use of age standardisation enables fair comparison of results from different studies (which may otherwise give misleading results if the age distribution of the populations being compared is different). The fact that only $23 \%$ of the identified studies met the inclusion criteria 
listed above suggests that the scientific quality of most of the papers published before 1999 on the epidemiology of childhood type 1 diabetes was quite variable.

At the time of publication, the paper by Onkamo and collaborators represented the most extensive analysis of global trends in the epidemiology of type 1 diabetes. They observed that the incidence reported per 100,000 children under 15 years of age (the same denominator has been used in this 'Then and now') showed a 60 -fold difference between Peru, represented by Lima, with the lowest annual incidence (0.5) and Finland with the highest annual incidence (30.3). The 25th percentile of the mean incidences was 6.4 , the 50 th percentile was 8.6 , and the 75 th percentile was 14.6. The annual change in incidence rate ranged from $-0.2 \%$ in Colorado, USA, to $9.5 \%$ in Leicestershire, UK. A statistically significant increase was seen in 24 of the 37 populations for which data were analysed. The global annual increase was found to be $3.0 \%$ per year during the time period from 1960 to 1996. The authors observed an inverse correlation between the increase in the country-specific incidence rate and the incidence indicating that the relative increase was more conspicuous in populations with a low incidence.

Onkamo and co-workers used the observed trends to predict the incidence of type 1 diabetes up to the year 2010 in the 37 populations studied. They applied both a linear and an exponential model for the predictions, the latter of which generated a higher rate in most populations (16 out of 18). As it turns out, in general, the actual increase has been greater than predicted by the exponential model. For example, the incidence in Finland in 2010 was predicted to be 50.2 based on the linear model and 57.9 according to the exponential model (Fig. 1); however, the actual incidence had risen to 64.2 in this country by 2005 [3]. Another example is Austria, which had an observed incidence of 7.8 for the time period 1979-1993. The incidence in 2010 was predicted to be 12.8 by the linear model and 14.6 by the exponential model, whereas the reported incidence in 2007 was 18.4 [4]. A recent European analysis of incidence trends of childhood type 1 diabetes in 20 population-based registers in 17 countries reported that all but two registers showed significant increases in incidence rates over the 15 years from 1989 to 2003 [5]. The annual increase ranged from 0.6 to $9.3 \%$, with an overall annual increase for all registers of $3.9 \%$. There were significant differences in the increases between age groups. The highest relative increase of $5.4 \%$ was observed among those diagnosed under the age of 5 years, followed by a $4.3 \%$ increase observed among those diagnosed from 5-9 years of age. The lowest relative increase of $2.9 \%$ was seen among those diagnosed from 10-14 years of age. Like Onkamo et al. [2], the European study [5] observed an inverse correlation between the annual increase in incidence and the incidence rate. The authors predicted that the incidence of type 1 diabetes will double

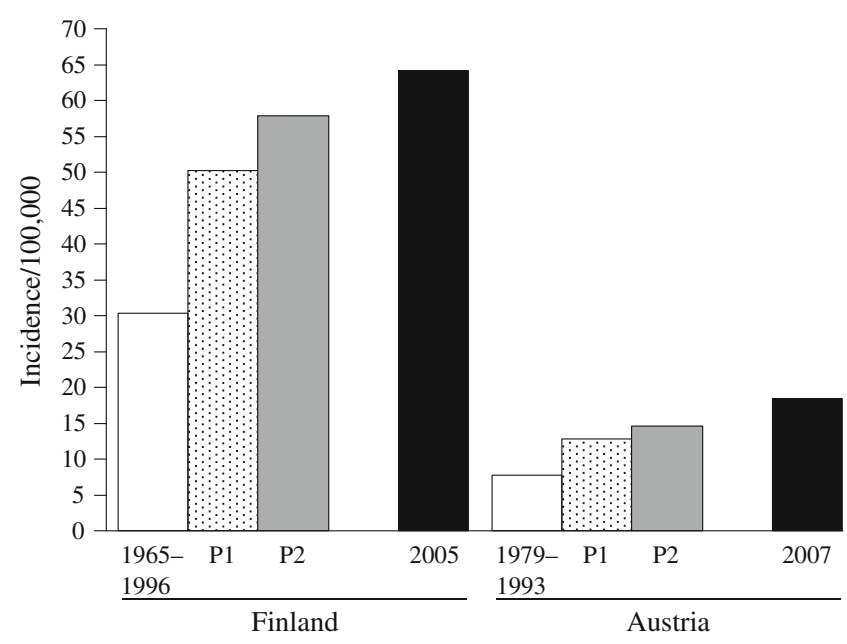

Fig. 1 Comparison between predicted incidence of childhood type 1 diabetes in Finland and Austria in 2010 [2] and observed incidence in Finland in 2005 [3] and in Austria in 2007 [4] showing that the observed rates exceeded the predicted ones in the previous 3-5 years. White bars, incidence 1965-1996 in Finland and 1979-1993 in Austria; dotted bars, predicted (P) incidence in 2010 in Finland and in Austria based on a linear model; grey bars, predicted incidence in 2010 in Finland and in Austria based on an exponential model; and black bars, observed incidence in Finland in 2005 and in Austria in 2007

among European children under the age of 5 years from the year 2005 up to 2020, and the number of children younger than 15 years with type 1 diabetes would increase by $70 \%$ over the same time period.

In 2008 Harjutsalo et al. provided data on time trends for type 1 diabetes in Finnish children [3]. Their analysis showed that the incidence of type 1 diabetes had increased from 31.4 in 1980 to 64.2 in 2005 . At $4.7 \%$, the relative annual increase was highest among those under the age of 5 years, while it was $2.7 \%$ among $5-9$-year-old children and only $1.7 \%$ in the oldest age group. The authors predicted

Table 1 Some hypotheses and exogenous factors proposed to contribute to the observed increase in the incidence of type 1 diabetes [11]

\begin{tabular}{|c|c|}
\hline Hypothesis/exogenous factor & Implied mechanism(s) \\
\hline The hygiene hypothesis & $\begin{array}{l}\text { A decreased microbial load in early } \\
\text { childhood affects the programming } \\
\text { of the immune system favouring } \\
\text { autoimmune and allergic responses }\end{array}$ \\
\hline The accelerator hypothesis & $\begin{array}{l}\text { Rapid growth and weight gain in } \\
\text { childhood induce beta cell stress }\end{array}$ \\
\hline The overload hypothesis & $\begin{array}{l}\text { Overload of the beta cells sensitises } \\
\text { them to immune damage }\end{array}$ \\
\hline The polio hypothesis & $\begin{array}{l}\text { Increase in early diabetogenic } \\
\text { enterovirus infections owing to } \\
\text { decreasing maternal antibody levels }\end{array}$ \\
\hline $\begin{array}{l}\text { Early introduction of } \\
\text { complex dietary proteins }\end{array}$ & $\begin{array}{l}\text { Increased gut permeability? } \\
\text { Unhealthy gut microbiome? }\end{array}$ \\
\hline Vitamin D deficiency & $\begin{array}{l}\text { Lacking immunomodulation } \\
\text { by vitamin } \mathrm{D}\end{array}$ \\
\hline
\end{tabular}


that the number of cases diagnosed before the age of 15 years would double from 2005 to 2020 . For age standardisation the two above-mentioned studies used an approach similar to that applied by Onkamo et al. [2].

Both the Finnish report and the European study point out that the fastest relative increase in incidence rate can be seen among children younger than 5 years of age. This leads to a younger average age at diagnosis. It also raises the issue of whether the increase in incidence observed in a series of studies from single centres, nationwide registers and international surveys represents a true increase or only reflects a decrease in age at diagnosis. There is no definite answer to this question, since the available data are contradictory. A Swedish analysis of incidence trends over a 16 -year period, including cases diagnosed before 35 years of age, suggested that there was no overall increase in the incidence rate but a significant decrease in the average age at diagnosis in both males and females [6]. In contrast, a recent study from the UK reported a significant increase in incidence both among those diagnosed before the age of 15 years and those diagnosed between the ages of 15 and 34 years during the time period from 1991 to 2005, although the increase was larger in the younger age group [7].

Another unresolved issue is whether there are any signs that the increase in incidence among children under the age of 15 years, seen in most populations studied, has started to level off. A study of the age-standardised incidence of childhood type 1 diabetes in Sweden over a 30-year period (1978-2007) showed that, over the first 22 years of the study period, the rate of type 1 diabetes increased substantially and shifted to a younger age at diagnosis. However, an analysis of the cumulative incidence by birth cohort suggested a reversed trend with an increasing age at diagnosis among those born in the year 2000 or later [8]. As reported from Norway, such trends may be transient [9].

The paper by Onkamo et al. [2] confirmed that the incidence of childhood type 1 diabetes increased worldwide during the latter part of the 20th century, considerably expanding the geographical coverage of previous studies. This study has been followed by a multitude of reports on incidence and incidence trends from various parts of the world. However, the reason why we have witnessed such an increase in the global incidence of childhood type 1 diabetes over the last 50-60 years has remained elusive. It is obvious that this cannot be due to a genetic effect, since the gene pool changes slowly over many generations. Accordingly, the increase in incidence and decrease in age at diagnosis must result from environmental changes and/or gene-environment interactions. An interesting observation, first reported by investigators in Finland [10], is that there has been a change in the distribution of predisposing HLA genotypes among children recently diagnosed with type 1 diabetes compared with those diagnosed 50-70 years ago, which has resulted in a reduction in the frequency of highrisk genotypes. This provides further support for an increasing role of environmental risk factors for diabetes over time. Over recent years a series of hypotheses and potential exogenous factors have been proposed to explain the increasing incidence of type 1 diabetes (Table 1). These include the hygiene hypothesis, the accelerator hypothesis, the overload hypothesis, the polio hypothesis, the early introduction of complex dietary proteins (e.g. cow's milk proteins and cereals) and vitamin D deficiency [11]. It is a true challenge for future epidemiological research to identify the factor(s) behind the increasing rate of type 1 diabetes and to design effective interventions aimed at turning the current trend around.

At this point it is important to consider the role of descriptive epidemiology in the context of type 1 diabetes. I think that there are at least two obvious challenges ahead. First, we need more data on the epidemiology of type 1 diabetes over the whole lifespan, especially regarding young adults. Second, it is important to continue to monitor the incidence of childhood type 1 diabetes to find out whether the increasing trend will level off or be reversed at some point and to generate data allowing the healthcare system to prepare for the burden conferred by an increasing number of patients with a longer disease duration. Descriptive epidemiology should, however, be complemented with 'aetiological' epidemiology aimed at identifying the causes behind the secular trends.

Duality of interest The author declares that there is no duality of interest associated with this manuscript.

Contribution statement The author was responsible for the conception and design of the manuscript, drafting the article and approval of the final version for publication.

\section{References}

1. Knip M, Korhonen S, Kulmala P et al (2010) Prediction of type 1 diabetes in the general population. Diabetes Care 33:1206-1212

2. Onkamo P, Väänänen S, Karvonen M, Tuomilehto J (1999) Worldwide increase in the incidence of type I diabetes-the analysis of the data on published incidence trends. Diabetologia 42:1395-1403, Erratum in: 43:685

3. Harjutsalo V, Sjöberg L, Tuomilehto J (2008) Time trends in the incidence of type 1 diabetes in Finnish children. Lancet 371:17771782

4. Schober E, Wakldhoer T, Rami B, Hofer S, the Austrian Diabetes Incidence Study Group (2009) Incidence and time trend of type 1 and type 2 diabetes in Austrian children 1999-2007. J Pediatr 155:190-193

5. Patterson CC, Dahlquist GG, Gyürüs E, Green A, Soltész G, the EURODIAB Study Group (2009) Incidence trends for childhood type 1 diabetes in Europe during 1989-2003 and predicted new cases 2005-20: a multicentre prospective registration study. Lancet 373:2027-2033 
6. Pundziute-Lyckå A, Dahlquist G, Nyström L et al (2002) The incidence of type I diabetes has not increased but shifted to a younger age at diagnosis in the 0-34 years group in Sweden 1983-1998. Diabetologia 45:783-791

7. Imkampe OK, Gulliford MC (2011) Trends in type 1 diabetes incidence rate in the UK in 0 - to 14-year-olds and in 15- to 34year-olds, 1991-2008. Diabet Med 28:811-814

8. Berhan Y, Waernbaum I, Lind T, Möllsten A, Dahlquist G, for the Swedish Childhood Diabetes Study Group (2011) Thirty years of prospective nationwide incidence of childhood type 1 diabetes: the accelerating increase by time trends to level off in Sweden. Diabetes 60:577-581
9. Aamodt G, Stene LC, Njølstad PR, Søvik O, Joner G, the Norwegian Childhood Diabetes Study Group (2007) Spatiotemporal trends and age-period-cohort modeling of the incidence of type 1 diabetes among children aged $<15$ years in Norway 1973-1982 and 1989-2003. Diabetes Care 30:884-889

10. Hermann R, Knip M, Veijola R et al (2003) Temporal changes in the frequencies of HLA genotypes in patients with type 1 diabetesindication of an increased environmental pressure? Diabetologia 46:420-425

11. Knip M, Veijola R, Virtanen SM, Hyöty H, Vaarala O, Åkerblom HK (2005) Environmental triggers and determinants of type 1 diabetes. Diabetes 54(suppl 2):S125-S136 